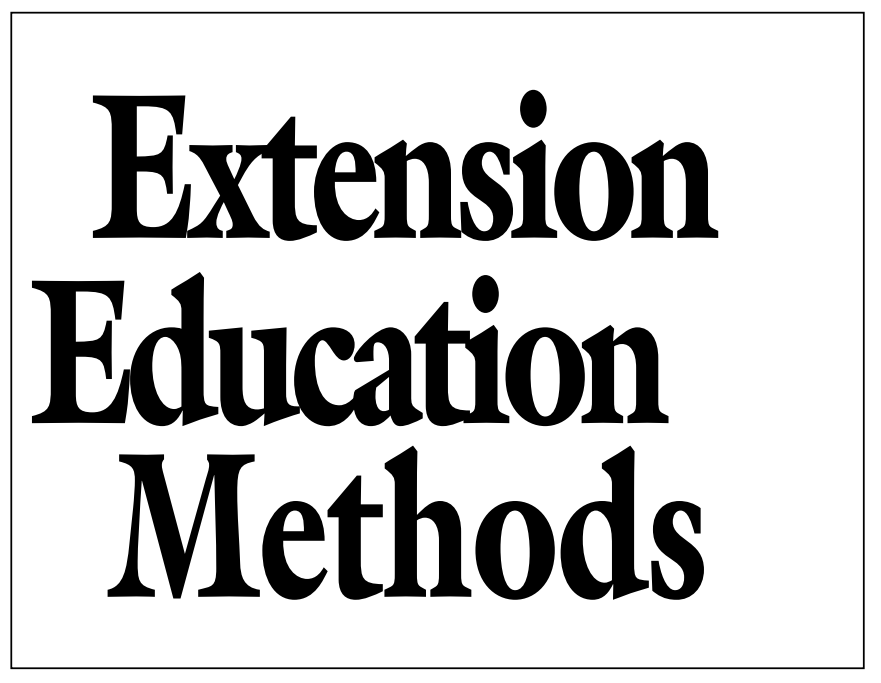

\section{Internet Video Access Appears Difficult for Oregon Extension Master Gardeners}

\author{
Ann Marie VanDerZanden ${ }^{1}$ and Bob Rost ${ }^{2}$
}

\begin{abstract}
Additional IndeX words. streaming video, multimedia, computer based training, distance learning, master gardener
\end{abstract}

SUmmary. Video clips are a type of interactive multimedia that are often incorporated in Internet based training, and recent studies have reported examples of how cooperative extension is beginning to use Internet technology, including video clips, as part of delivering educational programs online. A survey was designed to determine if Oregon State University Extension Master Gardener volunteers trained in 2001 were able to access a video clip online. Eighty percent of the recent trainees had access to or owned a computer and $93 \%$ of this group had access to the Internet. Yet, when asked to access the video clip online only $37 \%$ of the respondents were successful. This disparity suggests the need for a seamless interface between the multimedia component and the software required to access it. If the end user is unable to access the multimedia component, it is difficult to justify the additional resources required to develop these teaching tools.

$\mathrm{N}$ ew technologies, including the Internet, have resulted in a variety of relatively easy to create and easy to use media components that can be useful teaching tools. Examples include video and audio clips, animations and interactive text and graphics. Recent studies have shown that interactive multimedia, when compared to more traditional teaching methods can significantly increase learners' knowledge (Epstein and McGaha, 1999), higher order thinking skills (Taylor et al., 1997) and problem

${ }^{1}$ Associate professor and state coordinator Oregon Master Gardener Program, Department of Horticulture, Oregon State University, 4017 ALS Building, Corvallis, OR 97331-7304.

${ }^{2}$ Communications specialist, extension and experiment station communications, Oregon State University. solving skills (Bates, 1995). And to some extent, interactive multimedia allows the learner to control what they are learning and creates a more learner-directed education style which is compatible with adult learner preferences (Brown, 2001; Wilkes and Burnham, 1991).

Many of cooperative extension's educational programs are designed for adult audiences. Historically, most of these programs have been delivered face to face (Beaudin, 2000). However, recently some extension educators have started using new delivery methods and incorporating multimedia components such as audio and video clips, off-site links, email and listserves (Brown, 2001; DeCamp et al., 2001; Jeannette and Meyer, 2002; Lippert et al., 2000). Brown (2001) notes that for extension-type programs (programs that do not necessarily have a captive audience) to be successful, the participants must be engaged in the learning activity. Interactive multimedia may be one way to do that.

Although there is compelling evidence that interactive multimedia can enhance the educational experience for many learners (Brown, 2001), the question of accessibility of these multimedia components to the user is still unknown. Data collected in 2000 from a random stratified proportional sample of currently active Oregon Master Gardeners (MGs) showed that over $80 \%$ of the MGs own a computer and that $85 \%$ of this group are frequent Internet users (A.M. VanDerZanden, unpublished data). Yet VanDerZanden and Hilgert (2002) showed that the majority of Oregon MGs who completed an online training module through the MG program in 1999 were unable to access a video clip and two animations that were part of the module. This study was designed to evaluate the accessibility and usefulness of a 1.17 megabyte $(\mathrm{MB})$ video clip on seed germination, created using QuickTime (Apple Computer, Inc., Cupertino, Calif.) software and posted on the Oregon State University Northwest Gardening website (Oregon State University, 2001).

\section{Materials and methods}

In consultation with a survey specialist (L. Newton, Oregon State University Survey Research Center) a 19question survey [available online at http://osu.orst.edu/ extension/mg/videoclipevaluation (VanDerZanden, 2002)] was developed to determine accessibility of a 1.17-MB video clip on starting seeds. The video clip was available on the Northwest Gardening website at http://nwgardening.orst.edu (Oregon State University, 2001). Survey questions were designed to gather information on: computer ownership, type, and usage; Internet usage; modem connection; sound card capabilities; and whether or not the computer had a video player already loaded. Additionally there was a series of questions about the video and audio quality of the clip; if the video clip added to the information participants received in the plant propagation training session completed earlier in the year; and whether or not participants viewed any other video clips on the Northwest Gardening website.

The survey included stepwise instruction on how to complete the survey. If participants did not have access to a computer, the Internet, or their computer did not have a sound card (survey questions 1,3 , and 4 , respectively) they were asked to return the survey. After question 4 participants were directed to the video clip link on the Northwest Gardening website. In the event participants were unable to access the video clip using their existing computer configuration, links were provided to instructional websites on how to download three 


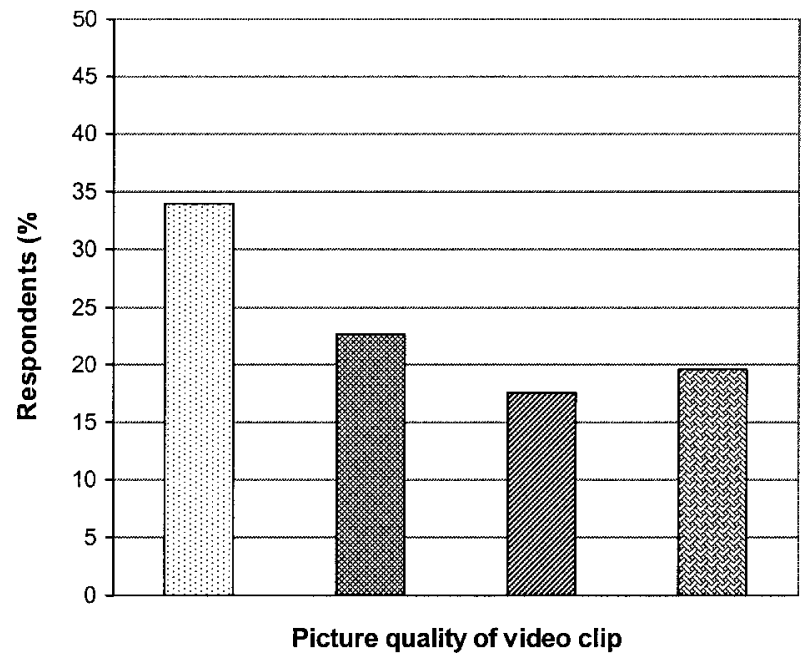

Fig. 1.Picture quality of a 1.17-megabyte video clip accessed online by trainees from the 2001 class of the Oregon Master Gardener program $(n=97)$.

commonplayerprograms, QuickTime, RealPlayer (RealNetworks, Inc., Seattle, Wash.), and Windows Media (Microsoft Corporation, Redmond, Wash.). If at this point participants were still unable to view the video clip they were instructed to go to the last survey question and then return the survey.

Before distribution of the survey, accessibility of the video clip was tested using three different Internet connections; a $56 \mathrm{~K}$ ( $\mathrm{K}=$ kilobits/s) phone modem, a cable modem and a $\mathrm{T}-\mathrm{l}$ line.

A random stratified proportional sample of 600 trainees, from a population of 852 , who completed the Oregon State University Master Gardener training program in 2001 were selected. The sample size was established using Perseus survey software (Perseus Development Corporation, Braintree, Mass.) with a power of $80 \%$ and alpha set to 0.05 . Three weeks after the survey was mailed, follow-up postcards were sent to nonrespondents. Data collection was completed October 2001. Descriptive statistics for each survey question were computed using Microsoft Excel (Microsoft Corporation, Redmond, Wash.).

\section{Results and discussion}

Of the 600 surveys sent to Oregon MGs, 326 usable surveys were returned for a response rate of $55 \%$. The returned surveys still represented a stratified proportional sample.

Eighty percent of the 326 respondents owned or had access to a computer. Of these 260 MGs, the majority (83\%) used their home computer most frequently while $11 \%$ used their computer at work most often, and 93\% (242 respondents) had access to the Internet via their most frequently used computer. A dial-up connection via phone line was the most common method of connecting to the Internet $(70 \%)$ while $20 \%$ used a highspeed connection [ $17 \%$ via cable; $3 \%$ via digital subscriber line (DSL)] and the remaining 10\% were either unsure or used a different type of connection. Of those who used a dial-up phone line connection, $70 \%$ had a $56 \mathrm{~K}$ modem, $17 \%$ were unsure of their modem speed, and the remaining categories $(28.8 \mathrm{~K}, 33.3 \mathrm{~K}$, or other $)$ accounted for $<7 \%$ each. When asked about a sound card in their computer, $85 \%$ noted they had one on the computer they used most often.

There were 208 respondents who had access to the Internet and a sound card and this group was directed to the Northwest Gardening website to access the video clip on starting seeds. Of the 208 MGs who went to the Northwest Gardening website, $46 \%$ were directed to download a player in order to view the video clip, $45 \%$ were not, and $9 \%$ of the MGs gave no response to this question. QuickTimeand RealPlayerwere the players downloaded most frequently, $44.8 \%$ and $39.6 \%$ respectively. In the end only $46.6 \%$ (97 respondents) were able to view the video clip, while over half $53.4 \%$ (111 respondents) were unable to view the video clip. The survey did not ask specifically why respondents were unable to view the clip but many provided written comments on the last question. The most common problems they noted were, inability to download a player, long down load times, and that their computer was not powerful enough to complete the function.

Of the 97 MGs that were able to view the video clip, 57\% felt the picture was "very clear" or "somewhat clear" (Fig. 1) and $80.2 \%$ thought the audio was "very clear" or "somewhat clear" (Fig. 2). Overall, 57\% thought viewing the video clip was "very easy" or "somewhat easy", while 38.1\% found it "somewhat difficult" or "very difficult" (Fig. 3). The final question about the video clip asked if the clip added to what the MGs had learned during the plant propagation training session they completed earlier in the year. Forty-five percent of the respondents thought the video clip "added a lot" or "added some" to the training, while $38 \%$ thought it "added very little" or "did not add at all" to what they had learned previously.

In addition to the video clip on starting seeds there were three other video clips on the same page of the Northwest Gardening website. Participants were not required to view these additional clips, but were asked in question 13 of the survey if they did. Of the $97 \mathrm{MGs}$ who viewed the seed-starting video clip, 39\% (38 respondents) viewed one or more of the other video clips; scaevola (Scaevola 'New Wonder') hanging baskets (33 respondents), excluding deer from the garden (29

Fig. 2. Audio quality of a 1.17-megabyte video clip accessed online by trainees from the 2001 class of the Oregon Master Gardener program $(\mathbf{n}=\mathbf{9 7})$.

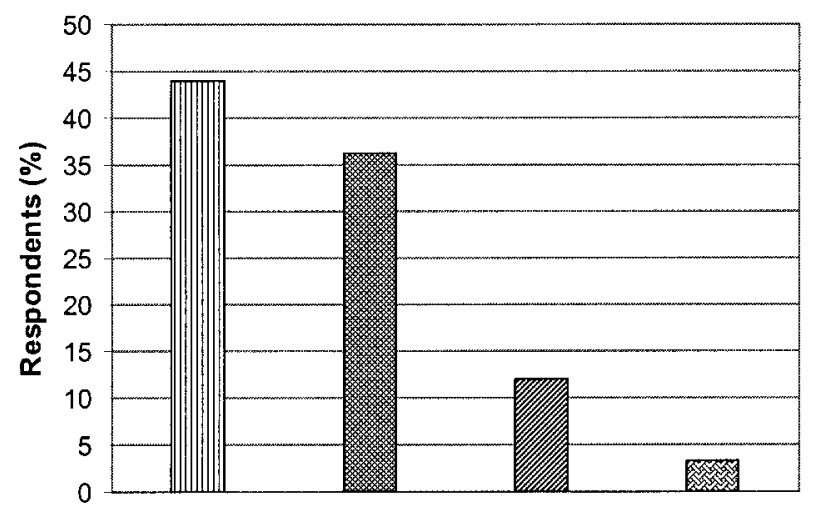

Audio quality of video clip 


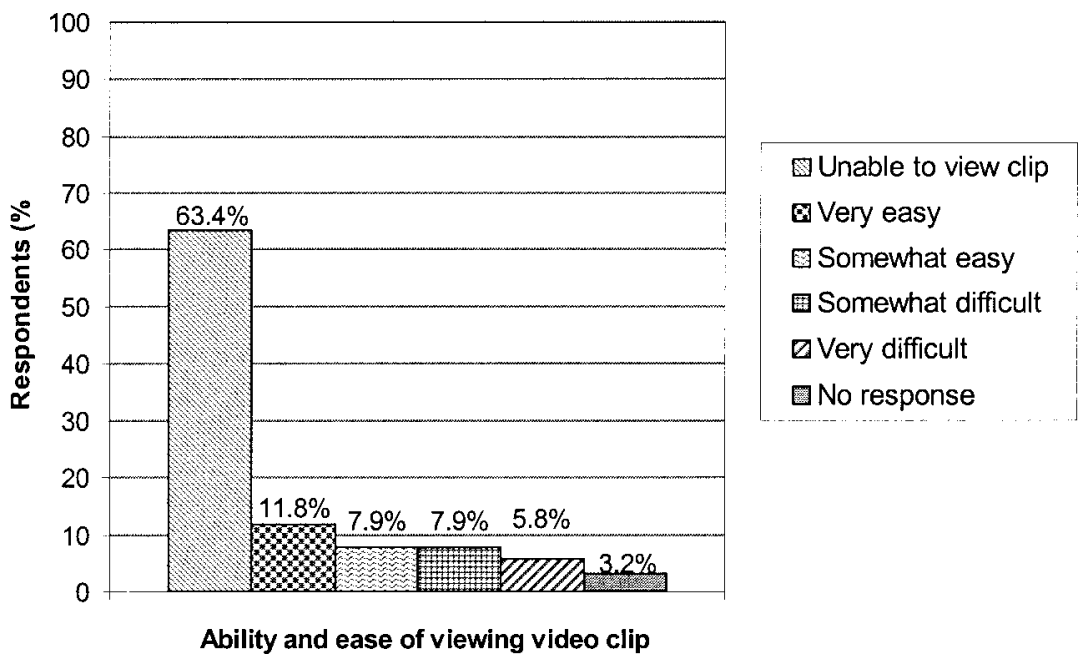

cost of delivering the training, and keeping costs lower by using the Internet is part of what makes this technology attractive. Regardless of how a multimedia component is made available, it is essential to include additional instruction to guide the user in accessing it.

Brown (2001) makes a strong case for the usefulness of video clips, audio clips, and animations in many educational settings. In VanDerZanden and Hilgert's (2002) article they clearly showed that the MGs who were completing an educational module online, gave their ability to access a video clip and two animations a highly negative ranking (4.1 on a Likert scale of 1 to 5 where $1=$ highly positive and $5=$ highly negative ranking) (Likert, 1932). As a result participants noted these three features did not enhance the quality of the online

Fig. 3. Ability and ease of viewing a 1.17-megabyte video clip accessed online by trainees from the 2001 class of the Oregon Master Gardener program $(n=326)$.

respondents), and a cooking segment on using fresh corn from the garden (16 respondents).

\section{Conclusions}

Although over $80 \%$ (261 respondents) of Oregon MGs who completed their training in 2001 own or have access to a computer, and $93 \%$ of those (242 respondents) have access to the Internet, only $37 \%$ (97 respondents) were able to view a 1.17-MB video clip via the Internet (Fig. 3). This suggests that although the technology is available to create interactive media, such as video clips, and a majority of Oregon MGs have the computer hardware capabilities to access such items, the majority are still unable to access them. The final question in the survey provided an opportunity for respondents to express their opinion about the video clip. There were a number of responses including comments from those who could access the clip and those who could not. General comments from those who viewed the clip were that it was easy to access, came across relatively clear although occasionally the transmission would break up, and that they would like to see more of these features.

However, there were far more comments from those respondents who were unable to view the clip. There was a range of comments, but some common themes emerged including; their computer did not have video player software installed, once prompted to download a player they did not understand the downloading instructions from the manufacturer, the software they tried to download was incompatible with their computer, and even that they received error messages that their computer did not have enough memory to process the function. A final group of comments were related to extremely long download times. The range reported by the respondents was from an hour and $28 \mathrm{~min}$ to $15 \mathrm{~min}$. Clearly these download times are unacceptable and many of them reported aborting the process after $15 \mathrm{~min}$.

The 1.17-MB size of the video clip used in this research is not exceptionally large for a video clip. Long download time is obviously a deterrent for some users and by using streaming video technology rather than a video clip the need to download the file would be avoided. Another alternative to downloading from the Internet would be to distribute the video clip to the MGs. Burning and distributing a CD-ROM adds to the course ( 4.0 on a 1 to 5 Likert scale). Unless the end user, in this case Oregon MGs, is able to access the multimedia component, the extensive time and resources required to develop such teaching tools may not be justified. As technology advances and computer interfaces become more seamless, these accessibility issues may be reduced or hopefully eliminated.

\section{Literature cited}

Bates, A.W. 1995. Technology, open learning and distance education. T.J. Press Ltd. Padstow, Cornwall, UK.

Beaudin, B. 2000. Delivering short seminars and workshops to farmers and ranchers with low bandwidth on-line technologies. J. Ext. 38(5). 14 Oct. 2002. http://www.joe.org/joe/2000october/rbl.html.

Brown, R. 2001. Thinking in multimedia: Research-based tips on designing and using interactive multimedia curricula. J. Ext. 39(3). 14 Oct. 2002. http://www.joe.org/joe/200ljune/ttl.html.

DeCamp, S., B. Richert, W. Singleton, N. Vines, and G. Slipher. 2001. Evaluating pork producers' acceptance of distance education media. J. Ext. 39(3). 14 Oct. 2002. http://www.joe.org/joe/200ljune/rb4.html.

Epstein, J. and (sic) McGaha. 1999. ATOD-TV: Evaluation of a multimedia program designed to educate the public about substance abuse. Computers in Human Behavior 15:73-83.

Jeannette, K.J. and M.H. Meyer. 2002. Online learning equals traditional classroom training for master gardeners. HortTechnology 12(1):148-156.

Likert, R. 1932. A technique for measurement of attitudes. Arch. Psychol. 140 .

Lippert, R.M., O. Plank, and R. Radhakrishna. 2000. Beyond perception: A pretest and posttest evaluation of a regional internet extension in-service training. J. Ext. 38(2). 14 Oct. 2002. http://www.joe.org/joe/2000april/ a2.html.

Oregon State University. 2001. Northwest gardening. 10. Dec. 2002. http://nwgardening.orst.edu.

Taylor, H., C. Renshaw, and M. Jensen. 1997. Effects of computer-based role playing on decision-making skills. J. Educ. Computing Res. 17:147164.

VanDerZanden, A.M. 2002. Video clip evaluation tool for the Oregon State University Master Gardener program. 10 Dec. 2002. http://osu.orst.edu/ extension $/ \mathrm{mg} /$ videoclipevaluation.

VanDerZanden, A.M. and C. Hilgert. 2002. Evaluating on-line training modules in the Oregon Master Gardener program. HortTechnology 12(2):297-299.

Wilkes, W.C. and B.R. Burnham. 1991. Adult learner motivations and electronic distance education. Amer. J. Distance Educ. 5:43-50. 